\title{
EVOLUCIÓN HISTÓRICA DE LA CONCEPCIÓN DE LA GESTIÓN DE RIESGOS DE DESASTRES: ALGUNAS CONSIDERACIONES
}

\author{
José Rosales-Veítia \\ Universidad Pedagógica Experimental Libertador - Instituto Pedagógico \\ de Caracas. Departamento de Ciencias de la Tierra, Venezuela
}

\begin{abstract}
Resumen: Así como la ciencia ha avanzado a pasos agigantados desde la segunda mitad del siglo XX, lo que hoy se conoce como gestión de riesgos ha pasado por una profunda transformación tanto conceptual como experimental, de allí que hoy por hoy se investigue principalmente desde las disciplinas sociales. En la actualidad, esta disciplina científica busca construir una equilibrada relación entre el ambiente y la sociedad humana. Existen países que están a la vanguardia en la temática, lo que permite vislumbrar que es viable para avanzar a un mundo mejor. El presente monográfico es una revisión documental sobre la concepción paradigmática de la gestión de riesgos de desastres a través de la historia y los postulados conceptuales básicos de la misma. Como conclusiones principales se obtiene: hoy por hoy, la gestión de riesgos ha pasado de atender de forma reactiva los eventos adversos a educar a la población para reducir los riesgos; además, para lograr buenos resultados en la gestión de riesgos de desastres es necesario empoderar a la población más expuesta a ocurrencia de eventos adversos.
\end{abstract}

Palabras clave: gestión de riesgos, evolución histórica, marcos de acción. 


\title{
Historical Evolution of the Conception of Disaster Risk Management: Some Considerations
}

\begin{abstract}
Just as science has advanced by leaps and bounds since the second half of the twentieth century, today what is known as risk management has gone through a profound conceptual as well as experimental transformation, which is why it is currently being investigated mainly from the social disciplines. Currently, this scientific discipline seeks to build a balanced relationship between the environment and human society. There are countries that have taken the lead on the issue, which allows us to see that it is viable to advance to a better world. The present monograph is a documentary review on the paradigmatic conception of risk management through history and the basic conceptual postulates of it. The main conclusions are: today, risk management has gone from reactively attending adverse events to educating the population to reduce risks; In addition, in order to achieve good results in risk management, it is necessary to empower the population most exposed to the occurrence of adverse events.
\end{abstract}

Keywords: Risk management, Historical evolution; Action frames.

\section{José Rosales-Veítia}

Profesor en Geografía e Historia, estudiante del subprograma de Especialización en Educación en Gestión de Riesgos, en el Departamento de Ciencias de la Tierra de la Universidad Pedagógica Experimental Libertador - Instituto Pedagógico de Caracas. Investigador adscrito al Programa de Estímulo al Investigador e Innovador (clase A-1) del Ministerio del Poder Popular para la Educación Universitaria, Ciencia y Tecnología, Venezuela.

Correo electrónico:andrew_rovei@hotmail.com

ORCID: https://orcid.org/0000-0002-0264-2715 


\title{
1. A manera de introducción
}

\author{
Debes asumir responsabilidad personal. No puedes cambiar las \\ circunstancias, las estaciones o el viento, pero te puedes cambiar a ti \\ mismo. Eso es algo que tienes a tu cargo (Jim Rohn).
}

Tal como puede observarse en el epígrafe, los eventos naturales que ocurren alrededor del planeta son eso: procesos propios del sistema terrestre. Por el contrario, las sociedades humanas son las que tienen la fragilidad ante la ocurrencia de estos, y la mezcla de ambos es lo que se termina llamando riesgos de desastres. No se puede predecir cómo, cuándo o dónde ocurrirá un evento adverso, pero sí se puede lograr reducir la vulnerabilidad ante ellos a través de la educación y la preparación, puesto que ambas son piedra fundamental de lo que hoy se conoce como gestión de riesgos de desastres (GRD), disciplina que ha pasado de ser muy limitada a todo un movimiento global que centra sus principios siempre con miras al desarrollo sostenible. Por ello, el presente monográfico tiene como objetivo identificar los cambios paradigmáticos que ha tenido la GRD desde 1960 hasta la actualidad.

A lo largo de la construcción del concepto que hoy se conoce como GRD se han hecho profundas transformaciones, desde comprender los eventos adversos como simples eventos físicos que azotan a la humanidad, a entender que la influencia que tiene el ser humano en el ambiente es elemento fundamental para constituir escenarios de riesgos. De allí que la Asamblea General de la Organización de las Naciones Unidas haya emanado desde hace más de cuarenta años documentos para buscar atender las consecuencias de los eventos adversos. Sin embargo, no es sino hasta la primera década del siglo XXI que se comprende que es más importante y viable atender desde la educación y formación de los ciudadanos, para así aumentar la resiliencia de la población y disminuir su vulnerabilidad. 


\section{La atención de los «desastres»}

El esbozo histórico inicia durante la década de los 60, periodo en el cual los eventos naturales eran sinónimos de desastres; incluso académicamente, los desastres eran trabajados por las ciencias físicas, incorporando estudios de disciplinas como sismología, vulcanología, geología, hidrología, climatología o meteorología (Gellert 2012). Estos estudios reafirmaban la postura: suceso de eventos naturales es igual a desastres, desvinculándose totalmente de los componentes sociales.

Así pues, ante la ocurrencia de eventos, los esfuerzos solo buscaban atender las consecuencias. Incluso la Asamblea General de las Naciones Unidas durante toda esta década «adoptó medidas como respuesta a diversos desastres severos», probablemente una de las más significativas para la época haya sido la resolución 2034 de 1965, titulada "Asistencia en casos de desastres», en la cual la Asamblea General solicitó a los Estados miembros que informaran al Secretario General acerca del tipo de asistencia que podían ofrecer en caso de emergencia (UNISDR s/f). Esta resolución es quizá la piedra fundamental que hizo al mundo congregarse para atender los riesgos, aunque concebido desde la atención ex post.

Por su parte, durante el periodo 1970-1980 se dio un importante avance desde la ingeniería en torno al tema de la GRD, pues comenzaron a hacerse planteamientos que se centraban en el cálculo de la pérdida, o como mejor podría decirse: en cualquier zona donde impacte una amenaza, los daños son medibles en el entorno físico, en la sociedad o en la economía (Gellert 2012). De allí comienza a vislumbrarse que el riesgo no es sinónimo de eventos naturales y comienza a comprenderse que los desastres están relacionados con impactos físicos extremos.

De esta manera, durante esta época comenzó a tratarse el tema de la prevención de desastres, y es así como la Asamblea General de las Naciones Unidas, entre los años 1970 y 1986, declaró a través de varias resoluciones que debía haber un proceso de planificación para reducir los desastres naturales, dos de las más representativas: a) resolución 2717 (1970), donde se establece que debe hacerse una planificación previa a los desastres para hacerles frente, pero que además debe involucrarse la tecnología para contenerlos; b) resolución 2816 (1971), donde se da la creación de la Oficina del Coordinador de las Naciones Unidas para el Socorro en Caso de Desastres (UNDRO, por sus siglas en inglés) (UNISDR s/f). A pesar de los considerables avances con respecto a la década anterior, en el fondo, las resoluciones de la ONU -incluso si se considera el componente social- se continuaban evaluando como parte de las consecuencias de los eventos, pero no como elemento fundamental para la construcción de los escenarios de riesgos, por lo cual se continuaron considerando únicamente como «desastres naturales», por tanto, los esfuerzos estaban orientados a contenerlos, y aunque comenzaban a surgir políticas dirigidas a la planificación previa a los desastres, la sociedad continuaba siendo reactiva ante la ocurrencia de los mismos. 
Cabe destacar que, aunque la ONU continuó durante los años 80 con la misma política del riesgo de los 70, académicamente surge el modelo conceptual prototipo del riesgo, donde se comienza a percibir:

$$
\text { Riesgo = Amenaza } * \text { Vulnerabilidad (Sanahuja 1999: 14) }
$$

Desde allí, las ciencias sociales se comienzan a ocupar del estudio de los desastres, centrándose con especial interés en los análisis de vulnerabilidad como factor determinante en la causa de los desastres, pues ya no se entiende la sociedad como un factor pasivo, sino activo en la formación del riesgo (Gellert 2012).

\section{De la atención de los «desastres» a la Gestión de Riesgos}

La década de 1990-1999 fue declarada por la Asamblea General de las Naciones Unidas como Decenio Internacional para la Reducción de los Desastres Naturales, el resultado fue la elaboración del primer documento a nivel mundial en su tipo que, según reza el mismo, estuvo orientado a dar mayor importancia a medidas que puedan adoptarse antes de que ocurran los desastres, en vez de la tendencia de respuesta ex post facto (Molin, 1997: 5). Aquí se plantea básicamente que los desastres son un problema no resuelto del desarrollo, pues se manifiestan sobre todo en zonas donde se ha dado un crecimiento demográfico no planificado. Surge entonces la postura de relación que existe entre lo natural y la organización estructural de la sociedad.

En este sentido y de acuerdo a Molin, el Decenio tuvo como objetivo: «Reducir por medio de una acción internacional concertada, especialmente en los países en vías de desarrollo, la pérdida de vidas, los daños materiales y trastornos sociales y económicos causados por los desastres naturales» (1997: 5). Es decir, los riesgos de desastres que se conciben en este periodo son meramente naturales y los esfuerzos de reducción giran en torno a ellos, por tanto, comienza a aplicarse el conocimiento, la tecnología y las experiencias obtenidas para vigilar y monitorear las amenazas, como estrategia para la reducción de la exposición ante ellas.

Asimismo, y bajo este enfoque del riesgo, en América Latina surge La RED (Red de Estudios Sociales en Prevención de desastres en América Latina), en el año 1992, como lugar de encuentro de opiniones de investigadores y de especialistas en el tema. Los desastres comienzan a estudiarse con mayor intensidad porque, contradictoriamente, durante el «Decenio» se presentaron con mayor frecuencia eventos de este tipo. De allí que el riesgo en este periodo haya sido entendido como lo define Mansilla: «Probabilidad que se manifieste una amenaza determinada sobre un sistema con un grado de vulnerabilidad dada» (2000: 18); las amenazas fueron entendidas como la posibilidad de ocurrencia de un evento físico que puede causar algún daño a la sociedad (Mansilla 2000); por su parte, la vulnerabilidad fue entendida como la propensión de una sociedad de sufrir daño 
y de encontrar dificultades de recuperarse posteriormente (Mansilla 2000). Estos planteamientos estaban fundamentados en lo que apareció por primera vez en el escenario mundial, y que estaba en contraposición a la atención del desastre: la gestión integral del riesgo, expuesta por Lavell como:

Proceso social complejo a través del cual se pretende lograr una reducción de los niveles de riesgo existentes en la sociedad y fomentar procesos de construcción de nuevas oportunidades de producción y asentamiento en el territorio en condiciones de seguridad aceptable (2000: 4).

Según se ha visto, durante el «Decenio» la ocurrencia de desastres se asocia con las amenazas de origen natural que afectan los asentamientos humanos, por tanto, los Estados deben tomar medidas orientadas a evaluar, monitorear y, en la medida de sus posibilidades, retener los desastres. Estos pasan a ser considerados como un problema no resuelto del desarrollo, introduce como respuesta a los mismos el desarrollo sostenible como alternativa, pues al utilizar los recursos naturales de forma responsable, la vulnerabilidad se reduce significativamente.

Así pues, una vez finalizado el «Decenio», la Asamblea General de las Naciones Unidas declara en el año 2000 la Estrategia Internacional para la Reducción de Desastres (EIRD), la cual inicia bajo la premisa que los desastres han causado grandes pérdidas en vidas humanas, destrucción de la infraestructura económica y social, pero además pone de manifiesto que han causado un impacto negativo en los ecosistemas, los cuales ya están frágiles por el desarrollo demográfico no planificado, por lo tanto se percibe que su ocurrencia es una amenaza para el desarrollo sostenible.

De esta manera, el objetivo de la EIRD según las Naciones Unidas fue: «Habilitar a las sociedades para resistir ante los peligros naturales y desastres tecnológicos ambientales relacionados con los mismos, con el propósito de reducir las pérdidas ambientales, humanas, económicas y sociales» (UNISDR 2001), en otras palabras, con lo aprendido en el «Decenio», la EIRD introdujo dos aspectos importantes en la GRD: primero, la importancia de sensibilizar a la población sobre la ocurrencia de eventos que no solo afectan a la sociedad, sino también el componente ambiental; segundo, los desastres no son naturales, son una construcción social que se origina por la relación de amenazas de diferentes tipos (naturales, socio-naturales, tecnológicas, biológicas) y una condición de vulnerabilidad ante la ocurrencia de eventos adversos.

Según lo planteado anteriormente, la EIRD fue diseñada para realizar una transformación profunda de paradigma, impulsando incluso un cambio conceptual, pasando de una protección contra los peligros a la gestión del riesgo; de allí que se desprendan dos campos de acción: 1) prevenir los desastres adoptando medidas para evitarlos, 2) reducir o limitar la severidad de los desastres a través de medidas adoptadas (UNISDR 2001).

Bajo estas premisas, laEIRD desarrolló nuevas concepciones delos conceptos fundamentales de los riesgos: a) peligros naturales: comprenden los fenómenos naturales que, por su naturaleza e impacto, pueden resultar amenazantes para una población; b) vulnerabilidad: grado de resistencia o susceptibilidad de un sistema 
socioeconómico con respecto al impacto de los peligros naturales (afectación del sistema socioeconómico) y desastres tecnológicos y ambientales (causados por los peligros naturales) relacionados con los mismos; y c) el riesgo: probabilidad que suceda un desastre (UNISDR, 2001).

Adicionalmente, durante este periodo, desde la academia se introdujeron conceptos importantes que fueron marcando un rumbo más claro con respecto al tema de riesgos en el mundo, configurando una concepción de la intervención antrópica en los mismos. La gestión de riesgos se definió según la EIRD como: "proceso de identificar, analizar y cuantificar las probabilidades de pérdidas y efectos secundarios que se desprenden de los desastres, así como las acciones preventivas, correctivas y reductivas correspondientes que deben emprenderse» (UNISDR 2004: 8).

Por su parte, el riesgo fue entendido por Mardones y Vidal como: «Probabilidad de ocurrencia en un lugar dado y en un momento determinado, de un fenómeno natural potencialmente peligroso para la comunidad y susceptible de causar daño a las personas y a sus bienes» (2001). La amenaza fue descrita por Ferrando como:

Procesos del medio ambiente físico que, por la gran magnitud que pueden o suelen alcanzar, son capaces de provocar cambios importantes en el paisaje o de alterar su condición de equilibrio de forma más o menos prolongada, según sea el tipo de proceso, el área afectada y la recurrencia (2003: 14).

De esta manera, puede apreciarse que los conceptos básicos de la GRD tuvieron un avance importante conceptualmente, pasando de ver los desastres como naturales para comprenderlos como un constructo social. Es allí cuando comienza a entenderse la importancia de la vulnerabilidad dentro de la GRD, Lavell (2000) la definió como: «Propensidad de sufrir daño, pero a la vez, una medida de las dificultades que enfrenta una sociedad para recuperarse del daño sufrido». Además, durante este periodo se introduce una expresión algebraica pedagógica para comprender la complejidad de la vulnerabilidad:

\section{Vulnerabilidad $=($ Susceptibilidad $/$ Sensibilidad Ambiental $) *$ Exposición} (Delgado 2002).

Se entiende entonces la susceptibilidad como potencial de cambio estructural y funcional que tiene un sistema ante una amenaza; la sensibilidad ambiental es la capacidad de darse cuenta, de prevenir, de estar alerta o de reaccionar; y la exposición es concebida como la situación espacio temporal de un individuo ante una amenaza o factor que puede inducir al cambio (Delgado 2002).

Igualmente, durante este periodo, la academia introdujo otro concepto que marcó el rumbo social de la gestión de riesgo: la resiliencia, que es definida por Delgado como: «Capacidad del individuo de reaccionar y recuperarse ante las adversidades, lo que implica un conjunto de cualidades que fomentan un proceso de adaptación exitosa y de transformación, a pesar de los riesgos y la propia adversidad» (2002). 
Así pues, bajo estos planteamientos se desarrolló la EIRD entre los años 2000 y 2007, donde se produjo una radical y profunda transformación paradigmática, que fue base fundamental para los cambios que vendrían en los años siguientes, pero desde entonces entendidos bajo la acción del hombre sobre las amenazas y la reducción de la vulnerabilidad.

\section{De la gestión de riesgos a la gestión del desarrollo}

Para el año 2005, la GRD comienza a trabajarse bajo la óptica del Marco de Acción de Hyogo 2005-2015, que implica un aumento de la resiliencia de las naciones y las comunidades ante los desastres, lo cual se abordó desde un enfoque sistemático que no se orientó a atender las consecuencias de los desastres, sino sus causas. Por tanto, puso especial énfasis en reducir la vulnerabilidad a las amenazas y los riesgos que conllevan, estableciendo la necesidad de aumentar la resiliencia en las naciones y las comunidades ante los desastres (UNISDR, 2005).

De esta manera, todas las metas y esfuerzos de este decreto estaban orientados con los Objetivos del Milenio. Desde allí comienza a concebirse la gestión de riesgos como un eje integrador, porque su reducción estaba conectada con cada uno de los objetivos: 1) Erradicar la pobreza extrema y el hambre: los pobres siempre son los más vulnerables, reducir los riesgos rompe con el circulo vicioso desastre-pobreza, y aumenta su resiliencia. 2) Lograr la enseñanza primaria universal: muchas escuelas no son construidas conforme a las normas y son estructuralmente muy deficientes; para lograr este objetivo habría que hacer las escuelas más seguras ante los desastres. 3) Fomentar la igualdad de género y el empoderamiento de la mujer: las mujeres son en mayor medida las más afectadas en situaciones de desastres, por eso ellas ocupan un lugar estratégico en las comunidades en la reducción de riesgos de desastres a través del desarrollo sostenible. 4, 5, 6) Reducir la mortalidad infantil, mejorar la salud materna y combatir el VIH/SIDA, el paludismo y otras enfermedades: muchas de las instalaciones sanitarias están mal construidas, sobre todo en los países en desarrollo, y aun en caso de desastres, si las edificaciones se mantienen en pie, su funcionamiento tiende a paralizarse, en especial los programas de control de enfermedades; por tanto, deben fortalecerse. 7) Garantizar la sostenibilidad del medio ambiente: reducir el riesgo es fundamental para adaptarse al cambio climático, en especial lo concerniente al acceso del agua potable, la reducción de la vulnerabilidad de los barrios marginales. 8) Fomentar una alianza mundial para el desarrollo: las alianzas sofisticadas congregan gobiernos, organizaciones no gubernamentales y el sector privado, por tanto, se deben hacer esfuerzos para transferir tecnologías a comunidades desfavorecidas para reducir sus riesgos (UIP y UNISDR 2010).

Según se ha visto, en este periodo la reducción de riesgos está inmerso en todo, es parte del componente social, y para lograr el desarrollo deben hacerse esfuerzos para reducir la vulnerabilidad y aumentar la resiliencia; se 
promueve enfáticamente una cultura de prevención y se involucra de forma activa a la comunidad porque se concibe que los desastres pueden reducirse considerablemente si la población está bien informada y empoderada del conocimiento.

Es así que los conceptos fundamentales fueron concebidos bajo esas premisas. Marcano y Cartaya definen el riesgo como: «Probabilidad que tiene un individuo o una población de ser afectado, según se tenga conocimiento de las características que condicionan el lugar de hábitat» (2010: 54). La amenaza fue entendida, según lo plantea el Marco de Acción de Hyogo, como: «Evento físico potencialmente perjudicial, fenómeno o actividad humana que puede causar pérdida de vidas o lesiones, daños materiales, grave perturbación de la vida social y económica o degradación ambiental, de origen natural o antrópica» (UNISDR 2005: 1). La vulnerabilidad fue entendida según Marcano y Cartaya como: «Factor de riesgo interno [...] que expresa la factibilidad de ser afectado por fenómenos naturales o de otro origen» (2010: 51). Igualmente, la resiliencia fue conceptualizada por el Marco de Acción de Hyogo como «capacidad de un sistema, comunidad o sociedad potencialmente expuesto a amenazas para adaptarse, resistiendo o cambiando» (UNISDR 2005: 4).

En la actualidad está en vigencia el Marco de Acción de Sendai para la Reducción de Riesgos de Desastres 2015-2030, el cual busca abordar la reducción de riesgos de desastres y aumentar la resiliencia, con especial énfasis en el desarrollo sostenible y la erradicación de la pobreza. Este marco aplica a riesgos de pequeña y gran escala, frecuentes y poco frecuentes, súbitos y de evolución lenta debido a amenazas naturales y de origen humano, amenazas y riesgos ambientales, tecnológicos y biológicos. Así, se tiene una nueva introducción al tema, pues se consideran ahora los riesgos biológicos que hasta la fecha no habían sido tomados en cuenta.

De acuerdo a lo planteado en el párrafo anterior, el Marco de Sendai tiene como objetivo, según lo plantea UNISDR:

Prevenir la aparición de nuevos riesgos de desastres y reducir los existentes, implementando medidas integradas e inclusivas de índole económica, estructural, jurídica, social, sanitaria, cultural, educativa, ambiental, tecnológica, política e institucional que prevengan y reduzcan el grado de exposición a las amenazas y la vulnerabilidad a los desastres, aumenten la preparación para la respuesta y la recuperación y refuercen de ese modo la resiliencia (2015: 12).

Asimismo, se plantea que el Estado tiene la responsabilidad primordial de prevenir y reducir los riesgos, por lo tanto, la gestión de riesgos debe estar orientada a proteger a las personas y sus bienes, la salud, los medios de vida, los bienes de producción, y los activos culturales y ambientales. Aunado a ello, se pone mayor énfasis en el empoderamiento de las autoridades locales y comunales, con una participación inclusiva; y, sobre todo, al momento de ocurrir los eventos, en la fase de recuperación, rehabilitación y reconstrucción, es fundamental prevenir nuevos desastres y reducir el riesgo. Así se hacen las comunidades más resistentes.

A la luz de los planteamientos anteriores, desde la academia hasta el momento se han planteado nuevas definiciones de riesgo, Guanoluiza expone: 
«Se define como riesgo a la probabilidad de pérdidas y daños en el futuro, estas pérdidas van desde las físicas hasta las sicosociales y culturales» (2017: 12). Las amenazas son entendidas por Vera y Albarracín como: «procesos externos e internos a un sistema, de origen natural, antrópico o socio natural, que interactúan con este y que tienen el potencial de inducir una transformación significativa en él, ya sea de manera lenta o súbita» (2017: 111). Igualmente, Vera y Albarracín describen la vulnerabilidad como:

[...] factor interno de riesgo y alude a las características de un sistema desde el punto de vista de su exposición, capacidad para anticipar, sobrevivir, resistir y recuperarse del impacto de una amenaza natural, antrópica o socionatural, que implica una combinación de factores que determinan el grado al que un sistema se encuentra en riesgo por un evento distinto e identificable de la naturaleza o de la sociedad (2017: 111).

De acuerdo a lo expuesto en el Marco de Sendai, la resiliencia es comprendida como:

[...] capacidad de un sistema, comunidad o sociedad expuestos a una amenaza para resistir, absorber, adaptarse y recuperarse de sus efectos de manera oportuna y eficaz, lo que incluye la preservación y la restauración de sus estructuras y funciones básicas (UNISDR 2015: 9).

\section{Buenas prácticas en gestión de riesgos}

Hay países que están a la vanguardia en el tema de la GRD, debido a que sus características físico-naturales así lo han exigido. De allí que puedan nombrarse las buenas prácticas que se han desarrollado en Japón, país que se encuentra ubicado en el Cinturón de Fuego del Pacífico, donde ocurre gran cantidad de actividad sísmica y volcánica, pero además es afectado por el paso de tifones; sin embargo, la experiencia adquirida por los desastres ocurridos, en conjunto con las políticas públicas en la materia y la constante educación y preparación de la sociedad, han hecho que su población tenga un alto nivel de resiliencia. Ya desde 1880 se han promovido leyes que giran en torno a la gestión de riesgos, y en la actualidad se promueven proyectos de conservación de la tierra, tienen tecnología de avanzada para el pronóstico del clima, han desarrollado una amplia red de comunicación de información de desastres y han preparado sistemas para la gestión de los mismos. Desde 2001 las investigaciones están dirigidas a reducir los riesgos comunitarios y a formar voluntarios para la gestión de riesgos y atención de emergencias; incluso desarrollan constantes simulacros en comunidades, empresas, oficinas de gobiernos, centros educativos y salud, a fin de aumentar la capacidad de respuesta de la población en caso de desastres (Dirección General de Administración de Desastres-Japón 2002).

Por su parte, América Latina y el Caribe es una de las regiones más propensas a la ocurrencia de desastres, la ONU expresa: «Desde el 2000, 152 millones de 
latinoamericanos y caribeños han sido afectados por 1205 desastres entre los que se cuentan inundaciones, huracanes y tormentas, terremotos, sequías, aludes, incendios, temperaturas extremas y eventos volcánicos» (Noticias ONU 2020).

En este sentido, al ser una región altamente amenazada, los países están trabajando en la reducción de sus vulnerabilidades y aumento de la resiliencia. Por ello, tal como describe Watanabe: «En América Latina, la gestión de desastres ha evolucionado de los enfoques verticales de respuesta y ayuda de emergencia hacia estrategias intersectoriales para la gestión del riesgo» (2015: 9).

En la región, existen países que se encuentran a la vanguardia en la GRD; para el caso de esta investigación se presentarán dos de ellos, pues a pesar de sus diferencias en cuanto a desarrollo económico se refiere, las acciones tomadas se fundamentan en la relación directamente proporcional: mayor educación es igual a menor vulnerabilidad.

Uno de los países punta de lanza de la GRD en la región es Cuba, puesto que por su ubicación geográfica es afectado continuamente por huracanes y tormentas tropicales. Por ello, la gestión de riesgos de desastres en este país es una prioridad; así pues, el Gobierno ha impulsado la descentralización de la gestión de riesgos, llegando incluso a las comunidades a través de los distintos frentes de asociación civil para la preparación y simulacros de evacuación en caso de desastres. La isla ha adoptado una serie de leyes que han apoyado la gestión de riesgos y han educado a los habitantes. Incluso, desde el año 2005 los estudios de reducción de riesgos de desastres son de carácter obligatorio y tienen como finalidad identificar, prevenir, estar preparados y dar respuesta eficaz y oportuna en caso de eventos comunitarios. Además de ello, han elaborado Sistemas de Alerta Temprana efectivos en cuanto a las amenazas naturales, además, han desarrollado una gran campaña de educación a la población a través de los medios de comunicación (Llanes 2008).

En Centro América, El Salvador ha pasado de considerarse un país de riesgo muy alto a riesgo alto; este avance ha sido motivado por la disminución de la vulnerabilidad (Unión Europea de Protección Civil y Ayuda Humanitaria et al. 2018). Es importante destacar — al igual que en los ejemplos de Japón o Cubaque El Salvador ha aplicado con mayor énfasis en las instituciones educativas las políticas relacionadas con la educación integral en GRD, promoviendo un análisis sistemático de las amenazas con los estudiantes, de las amenazas a las que se expone su institución educativa, así como su área circundante, lo que los lleva a un mayor nivel de sensibilidad ambiental, comprendiendo su exposición y vulnerabilidad estructural y social (Alpízar 2009). 


\section{A manera de conclusión}

\section{Figura 1: Línea de tiempo: evolución de la concepción del riesgo en función de los decretos de las Naciones Unidas}

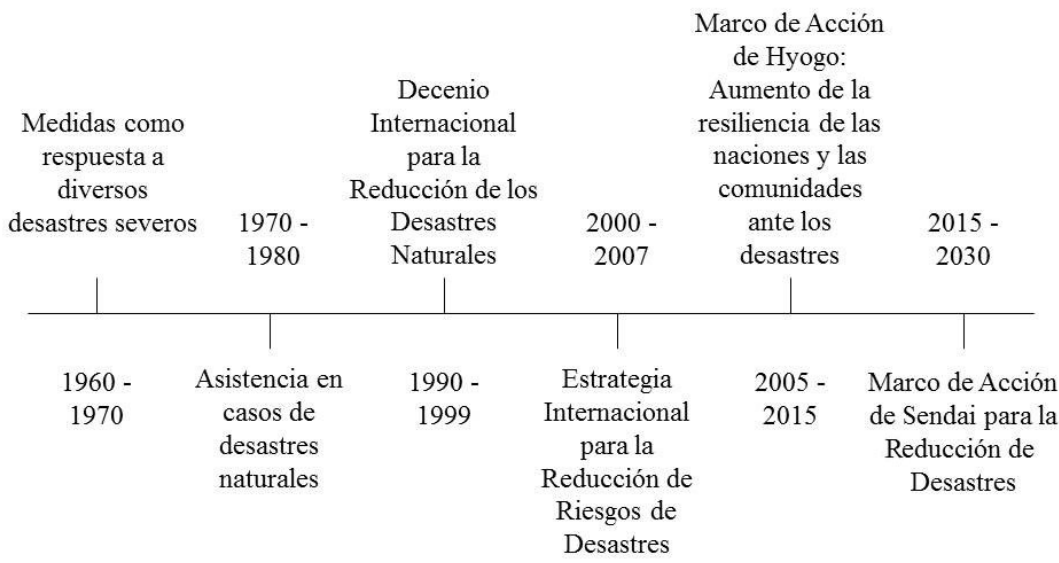

Fuente: Elaboración Propia

Primero, como puede observarse en la figura 1, la GRD ha pasado por un proceso de transformación paradigmática y conceptual que inició en la década de 1960 con la adopción de medidas como respuesta a diversos desastres severos, hasta llegar al Marco de Acción de Sendai Para la Reducción de Desastres. En la actualidad, una de las mayores premisas que se impulsa es que mientras mayor educada esté la población con respecto a las amenazas y los desastres, habrá una disminución sustancial de su vulnerabilidad.

Segundo, los riesgos son una construcción social y, por tanto, su concepción ha ido evolucionando a medida que los seres humanos han ido comprendiendo su implicación en los mismos; por lo que se ha tomado una posición donde ya no se es víctima en la ocurrencia de eventos, al contrario, se ha internalizado que la intervención antrópica sobre el espacio potencia la formación de escenarios de riesgos, por lo que es importante tomar medidas preventivas y correctivas en los mismos.

Tercero, los esfuerzos mundiales están comprometidos con la gestión integral de los riesgos de desastres, con la finalidad de reducir las pérdidas generadas no solo en las vidas humanas y materiales, sino que en la actualidad se considera la afectación en los ecosistemas, que se encuentran en estado de fragilidad por el cambio climático.

Cuarto, a medida que se evalúan cada uno de los decretos y marcos de acción de las Naciones Unidas luego de finalizados, ello permite esbozar las fortalezas de cada uno de esos decretos y marcos, pero en definitiva se busca determinar sus debilidades, a fin de fortalecer desde el aprendizaje para programas futuros. 
Quinto, en definitiva, a lo largo de la evolución de la concepción de la GRD (figura 1) se ha aprendido que los desastres no pueden predecirse ni tampoco contenerse, por tanto, debe trabajarse desde la educación formal, empleando a las instituciones educativas como pilares fundamentales para una gestión integral de los riesgos de desastres, a fin de enseñar a los estudiantes y sus familias a cómo identificar amenazas, recursos y vulnerabilidades, esto conlleva a un aumento sustancial de la resiliencia.

Sexto, la educación de la población y las comunidades, la formación de líderes locales, la implementación de sistemas de alerta temprana, el compartir saberes y experiencias, la divulgación de información con respecto al tema de los riesgos, permite, de acuerdo a las experiencias obtenidas, construir sociedades menos vulnerables ante la ocurrencia de eventos adversos.

Séptimo, la propensión de América Latina y el Caribe a la afectación por desastres ha sido una realidad que se evidencia desde 1990, relacionando el incremento de los eventos que han ocurrido en la zona con el cambio climático originado por las acciones antrópicas. Sin embargo, la aplicación real de estrategias de mitigación de riesgos ha conllevado a una disminución sustancial de los niveles de alto riesgo.

Octavo, los ejemplos de países como Japón, Cuba y El Salvador permiten vislumbrar que solo a través del empoderamiento del conocimiento y la formación para la atención de situaciones de emergencia se puede construir sociedades más resilientes ante los desastres.

Noveno, puede decirse que en la actualidad la GRD se encuentra en un paradigma social, donde las investigaciones están orientadas a construir, en conjunto con las comunidades, los inventarios de amenazas y recursos, los mapas de riesgos comunitarios, los planes de emergencia y respuesta, los protocolos de evacuación, con la intención que las comunidades se encuentren preparadas y empoderadas.

\section{Referencias}

Alpízar, M. (2009). Educación y Reducción de Riesgos y Desastres en Centroamérica: Gestión del Riesgo. San José de Costa Rica: CECC/SICA. https://ceccsica. info/sites/default/files/content/Volumen_50.pdf

Delgado, J. (2002). Hacia una planificación urbana para la reducción de riesgos ambientales. Vulnerabilidad urbana del área metropolitana de caracas. Urbana, 7(30): 25-41. http://190.169.94.12/ojs/index.php/rev_urb/ article/view/6019/5809

Dirección General de Administración de Desastres-Japón (2002). Administración de desastres en Japón. Caracas: Embajada de Japón. https://tinyurl.com/ et4dr8w

Ferrando, F. (2003). En torno a los desastres naturales: tipología, conceptos y reflexiones. Revista INVI, 18(47): 13-29. http://revistainvi.uchile.cl/index. php/INVI/article/view/388 
Gellert, G. (2012). El cambio de paradigma: de la atención de desastres a la gestión de riesgos. Boletín Científico Sapiens Research, 2(1): 13-17. https://tinyurl. com/4vzd2mtz

Guanoluiza, N. (2017). Evaluación De Amenazas, Riesgos y Vulnerabilidades en la Unidad Educativa Municipal «Milenio Bicentenario» del Distrito Metropolitano de Quito en el periodo Febrero-Julio 2017. Universidad Central de Ecuador: Trabajo de Grado Publicado.

Lavell, A. (2000). Sobre la Gestión del Riesgo: Apuntes hacía una Definición. En Garita, N. y Nowalski (Eds.). Del Desastre al Desarrollo Sostenible: El Caso de Mitch en Centroamérica. San José de Costa Rica: BID y CIDHS. www. desenredando.org/public/articulos/2000/dyd/DyD2000_mar-1-2002.pdf

Llanes, J. (2008). CUBA: Paradigma en la de riesgo de desastres. La Habana: Revista Unificada. https://www.preventionweb.net/publications/view/2558

Mansilla, E. (2000). Riesgo y Ciudad. Ciudad de México D.F.: UNAM. https://www. desenredando.org/public/libros/2000/ryc/RiesgoYCiudad-1.0.1.pdf

Marcano, A. y Cartaya, S. (2010). La Gestión de Riesgos de Desastres y el Uso de los Sistemas de Información Geográfica (SIG): Algunas Consideraciones. CONHISREMI, 6(3): 44-64. https://tinyurl.com/2faef5ny

Mardones, M. y Vidal, C. (2001). La zonificación y evaluación de los riesgos naturales de tipo geomorfológico: un instrumento para la planificación urbana en la ciudad de Concepción. EURE, 27(81). s/p. https://www. redalyc.org/articulo.oa?id=19608106

Molin, H. (1997). Decenio Internacional para la Reducción de los Desastres Naturales. En Lavell, A. (Comp.) Viviendo en riesgo. Comunidades vulnerables y prevención de desastres en America Latina (pp. 5-10). Naciones Unidas: Ginebra. https://www.desenredando.org/public/libros/1994/ver/ver_ final_nov-20-2002.pdf

Noticias ONU (3 de enero de 2020). América Latina y el Caribe: la segunda región más propensa a los desastres. Noticias ONU. https://tinyurl.com/3c4fxwpm

Sanahuja, H. (1999). El daño y la evaluación del riesgo en América Central: Una propuesta metodológica tomando como caso de estudio a Costa Rica. Trabajo de Grado Publicado. San José de Costa Rica: Universidad de Costa Rica. http://cidbimena.desastres.hn/docum/lared/libros/ EvaluacionRiesgoAmericaCentral-1.0.1.pdf

UIP y UNISDR (2010). Reducción del Riesgo de Desastres: Un Instrumento para alcanzar los Objetivos de Desarrollo del Milenio. Kit de Cabildeo para Parlamentarios. Ginebra: Unión Interparlamentaria. https://www.ipu.org/ file/1103/download

Unión Europea de Protección Civil y Ayuda Humanitaria et al. (2018). Índice de gestión de riesgos para América Latina y el Caribe: Actualización InformLAC 2018. Contribuyendo a una gestión más efectiva del riesgo de las crisis y los desastres en América Latina y el Caribe. UNICEF. https://www.unicef. org/lac/media/1601/file

UNISDR (s/f). Historia. UNDRR. https://www.eird.org/americas/we/historia. html 
UNISDR (2001). Marco de acción Para la aplicación de la Estrategia Internacional de Reducción de Desastres (EIRD). Ginebra: United Nations-UNISDR. https:// eird.org/esp/acerca-eird/marco-accion-esp.htm

UNISDR (2004). Gestión del riesgo. https://tinyurl.com/rz3vsnt9

UNISDR (2005). Marco de Acción de Hyogo 2005-2015: Aumento de la resiliencia de las naciones y las comunidades ante los desastres. Naciones Unidas: Ginebra. https://www.eird.org/cdmah/contenido/hyogo-framework-spanish.pdf

UNISDR (2015). Marco de Sendai para la Reducción del Riesgo de Desastres 20152030. Ginebra: United Nations. https://tinyurl.com/2ey6f6jz

Vera, J. y Albarracín, A. (2017). Metodología para el análisis de vulnerabilidad ante amenazas de inundación, remoción en masa y flujos torrenciales en cuencas hidrográficas. Ciencia e Ingeniería Neogranadina, 27(2): 109-136. http://www. scielo.org.co/pdf/cein/v27n2/0124-8170-cein-27-02-00109.pdf

Watanabe, M. (2015). Gestión del riesgo de desastres en ciudades de América Latina. Apuntes de InvestigAcción, 4(1): 1-17. https://tinyurl.com/5fktcss 